\title{
On the relation between fuzzy closing morphological operators, fuzzy consequence operators induced by fuzzy preorders and fuzzy closure and co-closure systems
}

\author{
Jorge Elorza $^{1 \mathrm{a}}$, Ramón Fuentes-González ${ }^{\mathrm{b}}$, Jean Bragard ${ }^{\mathrm{a}}$, Pedro Burillo ${ }^{\mathrm{b}}$ \\ ${ }^{a}$ Departamento de Física y Matemática Aplicada, Facultad de Ciencias, Universidad de \\ Navarra, P.O. Box 31008 Pamplona, Spain \\ ${ }^{b}$ Departamento de Automática y Computación, Universidad Pública de Navarra, Campus \\ de Arrosadía, P.O. Box 31006 Pamplona, Spain
}

\begin{abstract}
In a previous paper, Elorza and Burillo explored the coherence property in fuzzy consequence operators. In this paper we show that fuzzy closing operators of mathematical morphology are always coherent operators. We also show that the coherence property is the key to link the four following families: fuzzy closing morphological operators, fuzzy consequence operators, fuzzy preorders and fuzzy closure and co-closure systems. This will allow to translate important well-known properties from the field of approximate reasoning to the field of image processing.
\end{abstract}

Keywords: Fuzzy consequence operator, Fuzzy morphological operator, Fuzzy preorder, Fuzzy closure system, Lattice

\section{Introduction}

Mathematical morphology was born in the sixties from the collaborative work of G. Matheron and J. Serra $([33,40])$. Some years later, the algebraic

\footnotetext{
${ }^{1}$ Corresponding author. Departamento de Física y Matemática Aplicada, Facultad de Ciencias, Universidad de Navarra, P.O. Box 31008 Pamplona, Spain

Tel.:+34948425600; Fax:+34948425649

E-mail Address: jelorza@unav.es (J. Elorza)
} 
basis of mathematical morphology was introduced by H. Heijmans and C. Ronse ([31, 39]). The most important operators of morphological image processing were then analyzed and formalized (see for instance [30, 42]).

Extending mathematical morphology to fuzzy sets was proposed in the early nineties, by several teams independently $([1,9,18,19,41])$, and was then largely developed (see for instance $[6,10,16,17,20,32,34]$ ).

Generalizations of fuzzy mathematical morphology from different perspectives and interesting applications have been recently proposed (see for instance $[7,8,16,43,46])$.

In [27] the most used morphological operators have been studied in a new context: obtaining relevant information in fuzzy relational systems. The motivation for using binary relations as structuring element in this context will be explained in Section 2 because it requires some previous comments and definitions.

On another topic, consequence operators were introduced in classical logic by Alfred Tarski in 1930 ([44]). Tarski axiomatized the logic consequence concept trough consequence operators. Quoting Tarski, "a logic is just a set of propositions with a consequence operator".

The concept of fuzzy consequence operator was introduced in 1979 by J. Pavelka. He extends the concept of consequence operator defined by Tarski to fuzzy sets ([35]). Fuzzy consequence operators have been largely studied in the approximate reasoning context with different fuzzy logics (see for instance $[11,12,15,22,23,26,28,29,38])$.

Moreover, fuzzy consequence operators are closely related to the fuzzy preorders through the concept of coherence of an operator. Coherence concept was introduced in the early nineties by J.L. Castro and E. Trillas ([15]).

In short, fuzzy closing morphological operators and fuzzy consequence operators belong to the field of fuzzy mathematical morphology and approximate reasoning, respectively. Both fields require the algebraic framework of complete lattices where the above mentioned operators can be seen as closure operators $([2,3,4,47])$. These operators have also been studied as fuzzy modal operators (a thorough treatment from this perspective can be found in [37]).

The main purposes of the present paper are first to establish the interest- 
ing link between fuzzy mathematical morphology and approximate reasoning through these operators and then to connect both operators with the fuzzy preorders. Finally, all previous structures can be related with the classes of fuzzy closure and co-closure systems in the framework of complete lattices.

In Section 2, we recall some definitions, notations and results which will be used in subsequent sections.

In Section 3, we show that every fuzzy closing morphological operator induced by a fuzzy relation is a coherent fuzzy consequence operator. In particular, the coherence property will be the key to link all together the four following families: fuzzy closing morphological operators; fuzzy consequence operators; fuzzy preorders and fuzzy closure systems.

In Section 4, we analyze some properties of preorders induced from fuzzy closing operators and we show several characterizations of the main classes of fuzzy relations.

In Section 5, we embed fuzzy closing morphological operators in the classes of fuzzy consequence operators, fuzzy preorders and fuzzy closure systems.

Finally, in section 6 , we present some conclusions and propose directions for future works.

A large part of the results of sections 3 and 4 has been presented in the 14 th and 15th Spanish conferences on fuzzy logic and technologies $([24,25])$. In the present journal paper, we have included detailed proofs of these results in order to improve the readability of the paper.

\section{Preliminaries and basic definitions}

\subsection{Consequence operators and preorders}

As it is well established, in classical logic, consequence operators and preorders are strongly related. In order to facilitate the overall comprehension of the present paper, let us briefly recall these notions and their relationships. More details can be found in $[14,45]$.

From now on, $X$ will denote a nonempty (classical) universal set.

Let us recall (classical) preorder and consequence operators concepts. Given a non-empty universal set $X$ of logical propositions, as usual $\wp(X)$ 
represents the class of all subsets of $X$.

Definition 1. An operator $C: \wp(X) \longrightarrow \wp(X)$ is a Consequence Operator on $X$ if it satisfies the following properties:

(C1) $A \subset C(A)$ for all fuzzy subset $A \in \wp(X)$ (inclusion)

(C2) $A \subset B \Longrightarrow C(A) \subset C(B)$ for all $A, B \in \wp(X)$ (monotony)

(C3) $C(C(A))=C(A)$ for all $A \in \wp(X)$ (idempotence)

In the context of mathematical morphology, (C1) and (C2) are called extensive and increasing properties, respectively.

From a logical point of view, the previous axioms are interpreted in the following way:

If $X$ denotes a set of propositions, $A$ is a subset of axioms and $C(A)$ the theory generated by $A$ then

(C1) shows that the axioms are included into the theory.

(C2) shows that if the axiom set increases, the generated theory also increases.

(C3) shows that the theory is limited to the first step, i.e., if the theory is considered again as a starting set of axioms to generate a new theory, the latter is not increased.

Definition 2. A relation $R$ on $X$ (subset of $X \times X$ ) is a preorder if it satisfies the following properties:

(R1) $x R x \forall x \in X \quad$ (reflexivity)

(R2) If $x R y$ and $y R z$ then $x R z \forall x, y, z \in X \quad$ (transitivity).

From definitions 1 and 2, one obtains the classical relationship between preorders and consequence operators. Indeed, if we define a preorder $R$ on $X$ then automatically the corresponding consequence operator $C_{R}: \wp(X) \longrightarrow$ $\wp(X)$ induced by $R$ is defined as follows: $C_{R}(A)=\{b \in X ; \exists a \in A, a R b\}$.

Conversely, if $C$ is a consequence operator on $X$ then the induced relation by $C$ is given by $a R b \Longleftrightarrow b \in C(\{a\})$ and it is also a preorder.

Therefore, there exists a bijection between the class of all preorders on $X$ and the class of all consequence operators on $X$. It is clear that we must define a preorder as initial relation to obtain a logic in the classical sense (Tarski). That means that when $x R y$, we can say that $y$ is a consequence of $x$ in the sense of Tarski. 
In the present paper, $\left(L, \wedge, \vee, *, \rightsquigarrow_{*}, 0,1\right)$ will denote a residuated lattice with card $L>1$ (see [2]). $L$ will be the range of the memberships of the fuzzy subsets of $X$.

If there is no danger of confusion, we omit the subscript $*$ in $\rightsquigarrow_{*}$.

The structures where $L$ is the real unit interval $[0,1]$ are the most important for applications. In this case, the multiplication $*$ is a t-norm.

Let us recall that the multiplication $*$ in residuated lattices satisfies:

$$
\alpha *(\sup M)=\sup (\alpha * M) \text { for all } \alpha \in L, \text { for all } M \subset L
$$

This property generalizes a relevant property satisfied by left-continuous t-norms. In fact, if $*$ is also a t-norm, then it is usually called left-continuous or lower semicontinuous.

Let us remark that if $\left(L, \wedge, \vee, *, \rightsquigarrow_{*}, 0,1\right)$ is a residuated lattice and $L=$ $[0,1]$ then necessarily $*$ is a left-continuous t-norm.

Since there is a natural generalization of the concept of t-norm to lattices instead of the usual $[0,1]$ interval (see for instance [13]), we will refer to the multiplication $*$ also as a t-norm.

The extension of preorder concept to fuzzy $*$-preorder was already indicated in $([50])$ a few years after fuzzy set theory was proposed by L.A. Zadeh in $([49])$.

Definition 3. Let $*$ denote a t-norm. A fuzzy (binary) relation $R$ on $X$ (fuzzy subset of $X \times X)$ is called a $*$-fuzzy preorder if it satisfies:

(FR1) $R(x, x)=1 \forall x \in X \quad$ (reflexivity)

(FR2) $R(x, z) \geq R(x, y) * R(y, z) \forall x, y, z \in X \quad$ (*-transitivity).

If $R$ is also symmetric, i.e., $R(x, y)=R(y, x)$ for all $x, y \in X$, then it is called a fuzzy $*$-similarity, fuzzy $*$-indistinguishability or, equivalently, fuzzy *-equivalence. If $R$ is only reflexive and symmetric, it is called a fuzzy tolerance.

In 1979, J. Pavelka introduced the concept of fuzzy consequence operator ([35]) extending the concept of consequence operator in the sense of Tarski as a natural generalization to fuzzy sets. As usual, $L^{X}$ will represent the set of all functions from $X$ into $L$, this is, the set of all $L$-fuzzy subsets of $X$. 
Definition 4. An operator $C: L^{X} \longrightarrow L^{X}$ is a fuzzy consequence operator on $X$ if it verifies:

(FC1) $\mu \subset C(\mu)$ for all fuzzy subset $\mu \in L^{X} \quad$ (inclusion)

(FC2) $\mu_{1} \subset \mu_{2} \Longrightarrow C\left(\mu_{1}\right) \subset C\left(\mu_{2}\right)$ for all $\mu_{1}, \mu_{2} \in L^{X}$ (monotony)

(FC3) $C(C(\mu))=C(\mu)$ for all $\mu \in L^{X} \quad$ (idempotence)

Notice that, under (FC1) and (FC2) axioms, (FC3) may be also written equivalently as (FC3') $C(C(\mu)) \subset C(\mu) \forall \mu \in L^{X}$ that is a slightly weaker form of (FC3).

In 1991, J.L. Castro and E. Trillas proved ([15]) the following result: If $R$ is a fuzzy *-preorder then the operator $C_{R}^{*}$ between fuzzy subsets of $X$ given by the max-* Zadeh's compositional rule

$$
C_{R}^{*}(\mu)=\mu \circ^{*} R
$$

is a fuzzy consequence operator (induced by R), where

$$
\left(\mu \circ \circ^{*} R\right)(x)=\sup _{w \in X}\{\mu(w) * R(w, x)\}
$$

In 1999, J. Elorza and P. Burillo proved ([22]) that the converse of the previous result is also true: $R$ is a $*$-preorder if and only if $C_{R}^{*}$ is a fuzzy consequence operator. Therefore the only possibility to obtain fuzzy consequence operators from relations using Zadeh's max-* compositional rule is to work with $*$-preorders.

For the inverse process (inducing fuzzy preorders from fuzzy consequence operators), J.L. Castro and E. Trillas added the coherence axiom to the fuzzy consequence operator concept $([15])$.

Definition 5. Let $*$ denote a t-norm. An operator $C$ between fuzzy sets is called $*$-coherent if

$$
\mu(a) * C\left(\tilde{a}_{1}\right)(x) \leq C(\mu)(x)
$$

for all $\mu \in L^{X}$ and for all $(a, x) \in X \times X$, where $\tilde{a}_{1}(t)$ is the crisp membership function of the singleton $\{a\}$, that is, $\tilde{a}_{1}(t)=\left\{\begin{array}{ll}1 & \text { if } t=a \\ 0 & \text { if } t \neq a\end{array}\right.$.

It is proven in [15] that if $C$ is a $*$-coherent fuzzy consequence operator then the relation defined by

$$
R_{C}(x, y)=C\left(\tilde{x}_{1}\right)(y)
$$


is a fuzzy *-preorder (induced by $C$ ). It is also proven that the operator $C_{R}^{*}$ is always $*$-coherent.

In this paper, we will adopt the following notations for families of fuzzy relations and operators. We will denote by $\Gamma^{\prime}$ the class of all fuzzy relations on $X$ and for every t-norm $*, \Gamma^{*}$ the subfamily of all $*$-preorders.

$\Omega^{\prime}$ will be the class of all operators from $L^{X}$ into $L^{X}, \Omega$ will denote the family of fuzzy consequence operators and $\Omega_{r t}^{*}$ the subclass of all fuzzy consequence operators which induces a $*$ preorder by means of $(4), \tilde{\Omega}^{*}$ the subclass of all $*$-coherent fuzzy consequence operators and $\Omega_{p}^{*}$ will represent the subfamily of fuzzy consequence operators which are induced by a *preorder through Zadeh's max-* compositional rule, i.e.

$$
\Omega_{p}^{*}=\left\{C \in \Omega \mid \exists R \in \Gamma^{*}, C=C_{R}^{*}\right\}
$$

From previous paragraphs, it is clear the following chain of inclusions

$$
\Omega_{p}^{*} \subset \tilde{\Omega}^{*} \subset \Omega_{r t}^{*} \subset \Omega
$$

J. Elorza and P. Burillo proved ([22]) that every previous inclusion is strict:

$$
\Omega_{p}^{*} \varsubsetneqq \tilde{\Omega}^{*} \varsubsetneqq \Omega_{r t}^{*} \varsubsetneqq \Omega
$$

The construction of $C_{R}^{*}$ given by (1) can be generalized for any fuzzy relation $R$ then, for every t-norm $*$, we consider the function $\theta^{\prime *}: \Gamma^{\prime} \longrightarrow \Omega^{\prime}$ given by $\theta^{\prime *}(R)=C_{R}^{*}$ and $\theta^{*}$ will represent the restriction of $\theta^{\prime *}$ to the family $\Gamma^{*}$ of all $*$-preorders.

We also consider the function $\tilde{\theta}^{\prime}: \Omega^{\prime} \longrightarrow \Gamma^{\prime}$ given by $\tilde{\theta}^{\prime}(C)=R_{C}$ for all operator $C$ and $\tilde{\theta}$ will represent the restriction of $\tilde{\theta}^{\prime}$ to $\tilde{\Omega}$. Notice that $\tilde{\theta}^{\prime}$ and $\tilde{\theta}$ do not depend on the t-norm $*$. Let us remark that $\theta^{*}$ and $\tilde{\theta}$ are inverses of each other in the following sense: $\tilde{\theta} \circ \theta^{*}(R)=R$ for all $*$-preorder $R$ and $\theta^{*} \circ \tilde{\theta}(C)=C$ for all operator $C=C_{R}^{*}$ induced by an $*$-preorder.

These functions will be used in Section 5 .

\subsection{Morphological operators}

On another topic, the most used morphological operators have been studied in a new context: obtaining relevant information in fuzzy relational systems ([27]). In this context, the use of binary relations as structuring element is motivated as follows: 
In mathematical morphology, the effect of the basic dilation and erosion operators on an image $A$ is produced when this image $A$ interacts with certain family of translations of another fixed image $B$, called structuring element. For instance, for binary images $A \subset X$, where $X=\mathbb{R}^{2}$ or $X=\mathbb{Z}^{2}$, after assuming that $B \subset X$ is the structuring element, the dilation and the erosion of the set $A$ by $B$ can be expressed respectively by

$$
\delta_{B}(A)=\left\{x \in X \mid B_{x} \cap A \neq \emptyset\right\} \quad \text { and } \quad \varepsilon_{B}(A)=\left\{y \in X \mid \breve{B}_{y} \subset A\right\}
$$

where $B_{x}=\{x+b \mid b \in B\}$ and $\breve{B}_{y}=(-B)_{y}=\{y-b \mid b \in B\}$.

As it can be seen in the literature, morphological fuzzy dilation and erosion use a unary structuring element together with a translation operation (see for instance equations (7) in definition 2, page 2004 in [8]). Both dilation and erosion operators are related to the set of translations in $X$ and therefore, they are also related to the binary operation + in $\mathbb{R}^{2}$ or $\mathbb{Z}^{2}$.

At this point one can ask if it is possible to translate $\delta_{B}(A)$ and $\varepsilon_{B}(A)$ such that neither translations nor addition operation appear explicitly in the new definitions. This new formulation would be very useful for universal sets that do not possess a translations group and inner binary operations.

First let us propose new expressions of structuring elements and secondly we generalize them to universal sets $X$ that are not equal to $\mathbb{R}^{2}$ or $\mathbb{Z}^{2}$.

For any structuring element $B \subset X$, we define the binary relation $R_{B} \subset$ $X \times X$ as follows: $x R_{B} y \Longleftrightarrow y \in B_{x}$. From this relation, equations (5) can be translated by (6) and (7):

$$
\begin{gathered}
\delta_{R_{B}}(A)=\left\{x \in X \mid \exists y \in X:(y \in A) \wedge\left(x R_{B} y\right)\right\} \\
\varepsilon_{R_{B}}(A)=\left\{x \in X \mid \forall y \in X:\left(y R_{B} x\right) \Longrightarrow(y \in A)\right\}
\end{gathered}
$$

In a more general context that includes the fuzzy case, these equations can be rewritten as follows: given $R \in L^{X \times X}$ a fuzzy relation (structuring relation), dilation and erosion of $A \in L^{X}$ by $R$ are defined by 


$$
\begin{gathered}
\delta_{R}(A)(x)=(R \circ A)(x)=\sup _{y \in X}\{R(x, y) * A(y)\} \\
\varepsilon_{R}(A)(x)=\left(R^{o p} \triangleleft A\right)(x)=\inf _{y \in X}\{R(y, x) \rightsquigarrow A(y)\}
\end{gathered}
$$

for some t-norm $*$ and implication operator $\rightsquigarrow . R^{o p}$ denotes the inverse relation of $R, R^{o p}(x, y)=R(y, x)$

From a structuring fuzzy relation $R$, the most commonly used morphological operators (dilation, erosion, opening and closing) are then given respectively by $\delta_{R}(A)=R \circ A, \varepsilon_{R}(A)=R^{o p} \triangleleft A, \alpha_{R}(A)=R \circ\left(R^{o p} \triangleleft A\right)$ and $\varphi_{R}(A)=R^{o p} \triangleleft(R \circ A)$.

Both in the crisp and in the fuzzy cases, opening and closing operators with an isotropic structuring element are used in image processing to eliminate specific image details smaller than the structuring element. The global shape of the objects is not distorted.

In particular, a closing operator connects objects that are close to each other, fills up small holes and smooths out the object outline by filling up narrow gulfs. Meanings of near, small and narrow are related to the size and the shape of the structuring element.

Figure 1 shows an example where a set is transformed by a closing operator through a disk as structuring element. It is reproduced from the book "Morphological Image Analysis. Principles and Applications" by Pierre Soille. Springer, 1999, p.92, figure 4.5. (C)Springer-Verlag Berlin Heidelberg New York. It is taken with permission from Springer Science+Business Media and from the author Pierre Soille.

\subsection{Lattice theory and closure and co-closure systems}

Let us recall some basic notions and results on general lattice theory (for more details, see [5]).

Given a lattice $(L, \leq, \vee, \wedge)$ and given $S$ a subset of $L$, one says that $S$ is a sublattice of $L$ if $(S, \leq, \vee, \wedge)$ is a lattice, i.e., (i) $a \vee b \in S \forall a, b \in S$ and (ii) $a \wedge b \in S \forall a, b \in S$. In this case one can write $S \leq L$. 


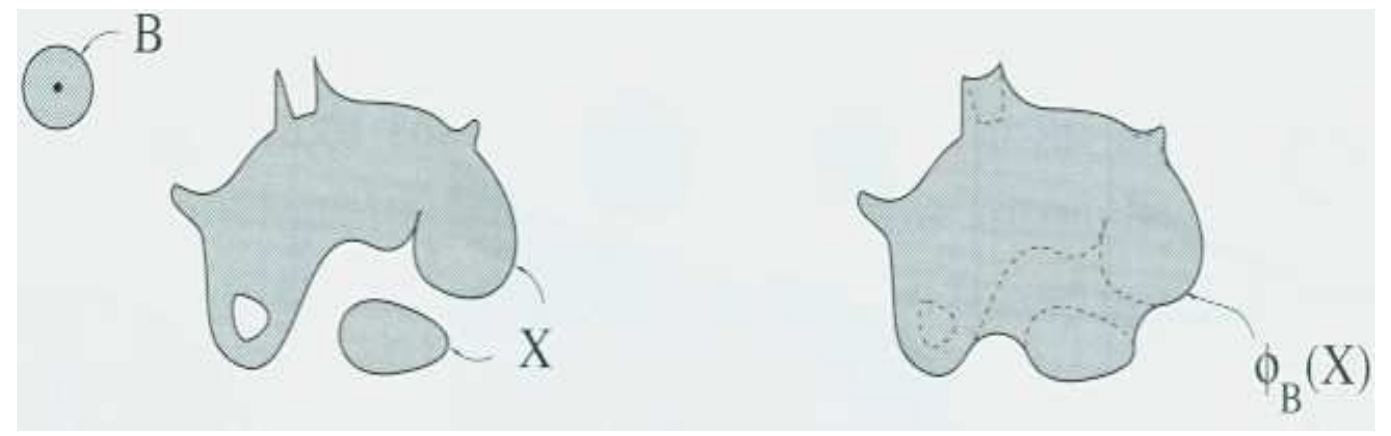

Figure 1: The effect of a closing operator on a non-smooth object (Reproduced from [42] with permission).

If only (i) is verified, then one says that $S$ is a $\vee$-sublattice (join-sublattice) of $L$ and one can write $S \leq_{\vee} L$. If only (ii) is verified, it means that $S$ is a $\wedge$-sublattice (meet-sublattice) of $L$ and one can write $S \leq_{\wedge} L$.

Any $\wedge$-semilattice $L$ which is complete and contains the greatest element is a complete lattice with a new supremum defined as follows $a \sqcup b=\bigwedge\{c \in$ $L \mid c \geq a \vee b\}$. Dually, any $\vee$-semilattice $L$ which is complete and contains the least element is a complete lattice with a new infimum defined as follows $a \sqcap b=\bigvee\{c \in L \mid c \leq a \wedge b\}$.

Let $\alpha: L \longrightarrow M$ be a function from a lattice $L$ into a lattice $M$. One says that $\alpha$ is isotone when $a \leq b$ implies $\alpha(a) \leq \alpha(b)$. One says that $\alpha$ is a $\vee$-morphism (join-morphism) if $\alpha(a \vee b)=\alpha(a) \vee \alpha(b)$ for all $a, b \in L$; a $\wedge$-morphism (meet-morphism) if $\alpha(a \wedge b)=\alpha(a) \wedge \alpha(b)$ for all $a, b \in L$; and a morphism when both conditions hold. As always, a morphism is called an isomorphism if it is a bijection. If there exists an isomorphism between $L$ and $M$, it means that $L$ and $M$ are isomorphic and one writes $L \cong M$; if there exists a join-morphism which is also a bijection, $L \cong_{\vee} M$ and if a meetmorphism which is also a bijection, $L \cong \wedge M$. One says that $\alpha$ is a dually morphism (or anti-morphism) if $\alpha(a \vee b)=\alpha(a) \wedge \alpha(b)$ and $\alpha(a \wedge b)=\alpha(a) \vee$ $\alpha(b)$ for all $a, b \in L$. Again, a dual morphism is called a dual isomorphism if it is a bijection. In the latter, one says that $L$ and $M$ are dually isomorphic and one can write $L \cong{ }^{d} M$. The following results hold: Any join-morphism between lattices is isotone; any meet-morphism is isotone; and finally, any isotone bijection with isotone inverse is a lattice isomorphism. 
Furthermore, it is well-known that if $L$ is a distributive complete lattice then the set $L^{X}$ of all functions from $X$ into $L$ (the set of all $L$-fuzzy subsets of $X)$ has the same structure. Thus, if $(\mathrm{L}, \leq, \vee, \wedge)$ is a complete lattice, so is the family $L^{X}$ with the following operations: $\mu_{1} \subset \mu_{2} \Longleftrightarrow \mu_{1}(x) \leq \mu_{2}(x) \forall x \in$ $X ;\left(\mu_{1} \vee \mu_{2}\right)(x)=\max \left(\mu_{1}(x), \mu_{2}(x)\right) ;\left(\mu_{1} \wedge \mu_{2}\right)(x)=\min \left(\mu_{1}(x), \mu_{2}(x)\right)$. The family $\Gamma^{\prime}=L^{X \times X}$ as family of fuzzy subsets of $X \times X$ is also a complete lattice with the previous operations between fuzzy relations. Analogously, the family $\Omega^{\prime}=\left(L^{X}\right)^{L^{X}}$ of all operators from $L^{X}$ into $L^{X}$ is another complete lattice with the following operations: $C_{1} \subset C_{2} \Longleftrightarrow C_{1}(\mu) \leq C_{2}(\mu) \forall \mu \in$ $L^{X} ;\left(C_{1} \vee C_{2}\right)(\mu)(x)=\sup \left(C_{1}(\mu)(x), C_{2}(\mu)(x)\right)$ and $\left(C_{1} \wedge C_{2}\right)(\mu)(x)=$ $\inf \left(C_{1}(\mu)(x), C_{2}(\mu)(x)\right)$.

Let us introduce some operations in the classes of fuzzy consequence operators and preorders. The class $\Omega$ of fuzzy consequence operators is not a sublattice of $\Omega^{\prime}$. However, $\Omega$ forms a complete lattice within $\Omega^{\prime}$ with the usual intersection and the following union: $C_{1} \sqcup^{\prime} C_{2}=\bigcap\left\{C \in \Omega \mid C \supset C_{1} \vee C_{2}\right\}$.

It is proven in [12] that the family $\Gamma^{*}$ of all fuzzy $*$-preorders is a complete $\wedge$-semilattice. Then $\Gamma^{*}$ with the usual $\subset, \wedge$ operations and the following $\sqcup^{*}$ : $R_{1} \sqcup^{*} R_{2}=\bigcap\left\{R \in \Gamma^{*} \mid R \supset R_{1} \vee R_{2}\right\}$ is a complete lattice.

Let us now discuss the notions and properties of closure and co-closure systems. A family $S$ of fuzzy subsets of $X$ is said to be a fuzzy closure system on $X$ if $\emptyset \in S, X \in S$ and $S$ is closed under arbitrary intersections. One denotes $C S(X)$ the class of all closure systems on $X$. A family $S$ of fuzzy subsets of $X$ is said to be a fuzzy co-closure system on $X$ if $\emptyset \in S, X \in S$ and $S$ is closed under arbitrary intersections and unions. One denotes by $C C S(X)$ the class of all co-closure systems on $X$.

In $C S(X)$ and $C C S(X)$, let us consider the analogous operations $\sqcup^{\prime}$ and $\sqcup: S_{1} \sqcup^{\prime} S_{2}=\bigcap\left\{S \in C S(X) \mid S \supset S_{1} \vee S_{2}\right\}$ and $S_{1} \sqcup S_{2}=\bigcap\{S \in C C S(X) \mid$ $\left.S \supset S_{1} \vee S_{2}\right\}$.

For every operator $C: L^{X} \longrightarrow L^{X}$, we denote by $\alpha^{\prime}(C)$ the family of fixed fuzzy subsets by $C$, i.e. $\alpha^{\prime}(C)=\left\{\mu \in L^{X} \mid C(\mu)=\mu\right\}$. $\alpha$ indicates the restriction of $\alpha^{\prime}$ to $\Omega$. The function $\alpha$ is a lattice dual isomorphism between complete lattices $\left(\Omega, \sqcup^{\prime}, \wedge\right)$ and $\left(C S(X), \sqcup^{\prime}, \wedge\right)$. Thus $\Omega \cong d C S(X)[11$, 47]. Moreover the inverse function of $\alpha$ is $\alpha^{-1}(S)=C_{S}$, where $C_{S}(\mu)(x)=$ $\inf _{\nu \in S, \nu \supset \mu}\{\nu(x)\}$. 
Let us recall that, given a left-continuous (lower semicontinuous) t-norm $*, \rightsquigarrow_{*}$ is the notation for the residuated implication induced by the t-norm $*$, i.e. $\alpha \rightsquigarrow_{*} \beta=\sup \{w \in L / \alpha * w \leq \beta\}$. It is sometimes called the pseudoinverse application of $*$.

Finally, the following functions $I^{*}$ and $\Im^{*}$ introduced in [12] will be used in Section 5. $I^{*}: \wp\left(L^{X}\right) \rightarrow \Gamma^{*}$ given by $I^{*}(S)(a, b)=\inf _{\nu \in S}\left\{\nu(a) \rightsquigarrow_{*} \nu(b)\right\}$ $\forall a, b \in X . I^{*}(S)$ is called the $*$-implication relation induced from $S$.

$\Im^{*}(R): \Gamma^{\prime} \rightarrow C C S(X)$ given by $\Im^{*}(R)=\left\{\mu \in L^{X} \mid \mu(a) * R(a, b) \leq\right.$ $\mu(b) \forall a, b \in X\}$. If $\mu \in \Im^{*}(R)$, it is called a $*$-true fuzzy set or a closed under modus ponens fuzzy set or equivalently a valuation $*$-compatible with $R$.

The general properties of $I^{*}$ and $\Im^{*}$ functions are summarized as follows (i) $I^{*}$ and $\Im^{*}$ are antitone functions $\left(I^{*}\left(S_{1}\right) \subset I^{*}\left(S_{2}\right)\right.$ if $S_{2} \subset S_{1}$ and $\Im^{*}\left(R_{1}\right) \subset$ $\Im^{*}\left(R_{2}\right)$ if $\left.R_{2} \subset R_{1}\right)$.

(ii) $S \subset \Im^{*}\left(I^{*}(S)\right) . R \subset I^{*}\left(\Im^{*}(R)\right)$.

(iii) $I^{*}\left(\Im^{*}(R)\right)=R_{*}^{c}$, where $R_{*}^{c}$ denotes the smallest $*$-fuzzy preorder which contains $R$.

(iv) $\Im^{*}\left(R_{1}\right)=\Im^{*}\left(R_{2}\right)$ if and only if $R_{1 *}^{c}=R_{2 *}^{c}$

(v) If $R$ is a $*$-fuzzy preorder then $R=I^{*}\left(\Im^{*}(R)\right)$, this is, $I^{*} \circ \Im^{*}$ is the identity mapping on $\Gamma^{*}$. Therefore the restriction of $\Im^{*}$ to $\Gamma^{*}\left(\Im^{*} \mid \Gamma^{*}\right.$ : $\left.\Gamma^{*} \longrightarrow C C S(X)\right)$ is one to one and the restriction of $I^{*}$ to $\operatorname{CCS}(\mathrm{X})\left(I^{*}\right.$ : $\left.C C S(X) \longrightarrow \Gamma^{*}\right)$ is onto.

Next, let us recall three properties of t-norms, infimum and residuated implications which will be used in Section 4:

$$
\begin{gathered}
x * \inf _{i \in I}\left\{y_{i}\right\} \leq \inf _{i \in I}\left\{x * y_{i}\right\} \\
x *\left(y \rightsquigarrow_{*} z\right) \leq y \rightsquigarrow_{*}(x * z) \\
y_{1} \leq y_{2} \text { implies } x \rightsquigarrow_{*} y_{1} \leq x \rightsquigarrow_{*} y_{2}
\end{gathered}
$$

The following property will be used in Section 3:

$$
x_{1} \leq x_{2} \text { implies } x_{2} \rightsquigarrow_{*} y \leq x_{1} \rightsquigarrow_{*} y
$$




\section{Coherence of fuzzy closing morphological operators}

The concept of fuzzy closing operator $\varphi_{R}^{*}$ induced by a fuzzy relation is given by

$$
\varphi_{R}^{*}(\mu)=R^{o p} \triangleleft_{\rightsquigarrow *}\left(R \circ^{*} \mu\right)
$$

where

$$
\left(R \circ^{*} \mu\right)(x)=\sup _{w \in X}\{R(x, w) * \mu(w)\}
$$

and

$$
\left(R \triangleleft_{\rightsquigarrow_{*}} \mu\right)(x)=\inf _{w \in X}\left\{R(x, w) \rightsquigarrow_{*} \mu(w)\right\}
$$

It is well-known that $\varphi_{R}^{*}$ is a closure operator and therefore a fuzzy consequence operator. In this section, we will prove that $\varphi_{R}^{*}$ is always an $*$-coherent operator as fuzzy consequence operator.

First, let us prove two preliminary results.

Lemma 1. Let $R$ be any fuzzy relation on $X, \mu \in L^{X}$ and $x \in X$, then:

$$
\varphi_{R}^{*}(\mu)(x)=\inf _{w \in X}\left\{R^{o p}(x, w) \rightsquigarrow_{*} C_{R^{o p}}^{*}(\mu)(w)\right\}
$$

Proof. It is straightforward from (2), (14) and (15).

Lemma 2. Let $R$ be any fuzzy relation on $X$ and $a, x \in X$, then:

$$
C_{R}^{*}\left(\tilde{a}_{1}\right)(x)=R(a, x)
$$

Proof. Given $a, x \in X$ :

$$
C_{R}^{*}\left(\tilde{a}_{1}\right)(x)=\left(\mu \circ^{*} R\right)(x)=\sup _{w \in X}\left\{\tilde{a}_{1}(w) * R(w, x)\right\}
$$

Observe that this supremum is obtained when $w=a$. Then, we have

$C_{R}^{*}\left(\tilde{a}_{1}\right)(x)=\tilde{a}_{1}(a) * R(a, x)=1 * R(a, x)=R(a, x)$ 
Theorem 1. Let $R$ be any fuzzy relation on $X$. Then the fuzzy closing operator $\varphi_{R}^{*}: L^{X} \longrightarrow L^{X}$ induced by $R$ by means of (14) is a $*$-coherent fuzzy consequence operator .

Proof. It is enough to prove the coherence condition. Given $\mu \in L^{X}$ and $a, x \in X$. From Lemma 1:

$$
\begin{gathered}
\mu(a) * \varphi_{R}^{*}\left(\tilde{a}_{1}\right)(x)= \\
\mu(a) * \inf _{w \in X}\left\{R^{o p}(x, w) \rightsquigarrow_{*} C_{R^{o p}}^{*}\left(\tilde{a}_{1}\right)(w)\right\}
\end{gathered}
$$

From Lemma 2:

$$
\begin{gathered}
\mu(a) * \varphi_{R}^{*}\left(\tilde{a}_{1}\right)(x)= \\
\mu(a) * \inf _{w \in X}\left\{R^{o p}(x, w) \rightsquigarrow_{*} R(w, a)\right\}= \\
\mu(a) * \inf _{w \in X}\left\{R(w, x) \rightsquigarrow_{*} R(w, a)\right\}
\end{gathered}
$$

By properties (10) and (11) one gets:

$$
\begin{gathered}
\mu(a) * \varphi_{R}^{*}\left(\tilde{a}_{1}\right)(x) \leq \\
\inf _{w \in X}\left\{\mu(a) *\left(R(w, x) \rightsquigarrow_{*} R(w, a)\right)\right\} \leq \\
\inf _{w \in X}\left\{R(w, x) \rightsquigarrow_{*}(R(w, a) * \mu(a))\right\}
\end{gathered}
$$

Observe that

$$
\begin{gathered}
C_{R^{o p}}^{*}(\mu)(w)=\sup _{u \in X}\{R(w, u) * \mu(u)\} \geq \\
R(w, a) * \mu(a)
\end{gathered}
$$

Finally, by property (12):

$$
\begin{gathered}
\mu(a) * \varphi_{R}^{*}\left(\tilde{a}_{1}\right)(x) \leq \\
\inf _{w \in X}\left\{R(w, x) \rightsquigarrow_{*} C_{R^{o p}}^{*}(\mu)(w)\right\}=\varphi_{R}^{*}(\mu)(x)
\end{gathered}
$$

Therefore $\mu(a) * \varphi_{R}^{*}\left(\tilde{a}_{1}\right)(x) \leq \varphi_{R}^{*}(\mu)(x)$ and $\varphi_{R}^{*}$ is $*$-coherent. 


\section{Fuzzy relations induced by fuzzy closing morphological opera- tors}

We have proven in the previous section that the fuzzy closing operator $\varphi_{R}^{*}$ induced by a relation $R$ by means of (14) is $*$-coherent. As a consequence the relation induced by the fuzzy consequence operator $\varphi_{R}^{*}$ by means of (4) is a $*$-preorder $\left(\varphi_{R}^{*} \in \tilde{\Omega}^{*} \subset \Omega_{r t}^{*}\right)$.

In this section, we introduce a characterization of such induced relation in order to analyze some properties.

Let us recall that the $\circ^{*}, \triangleleft_{\rightsquigarrow_{*}}$ compositions and the residuated implication $\rightsquigarrow_{*}$ of two fuzzy binary relations $R$ and $S$ on $X$ are defined by

$$
\begin{gathered}
\left(R \circ^{*} S\right)(x, y)=\sup _{w \in X}\{R(x, w) * S(w, y)\} \\
\left(R \triangleleft \rtimes_{*} S\right)(x, y)=\inf _{w \in X}\left\{R(x, w) \rightsquigarrow_{*} S(w, y)\right\}
\end{gathered}
$$

and

$$
\left(R \rightsquigarrow_{*} S\right)(x, y)=R(x, y) \rightsquigarrow_{*} S(x, y)
$$

Lemma 3. Let $R$ be any fuzzy relation on $X$ and let us denote $C=\varphi_{R}^{*}$. Then the *-preorder $R_{C}$ induced by $C$ by means of (4) is such that

$$
R_{C}=\left(R^{o p} \triangleleft_{\rightsquigarrow_{*}} R\right)^{o p}
$$

Proof. Using Lemmas 1 and 2 one gets:

$$
\begin{gathered}
\left(R^{o p} \triangleleft_{\rightsquigarrow_{*}} R\right)^{o p}(x, y)=\left(R^{o p} \triangleleft_{\rightsquigarrow_{*}} R\right)(y, x)=\inf _{w \in X}\left\{R(w, y) \rightsquigarrow_{*} R(w, x)\right\}= \\
{\left[R^{o p} \triangleleft_{\rightsquigarrow_{*}}\left(R \circ^{*} \tilde{x}_{1}\right)\right](y)=R_{C}(x, y)}
\end{gathered}
$$

Remark 1. Let us note that given any fuzzy relation $R, R^{o p} \triangleleft_{\rightsquigarrow_{*}} R$ is always $*$-transitive even though it does not involve the o operator. 
In the following, we will use the identity relation $I$ on $X$, i.e. $I(x, y)=$ $\left\{\begin{array}{ll}1 & \text { if } x=y \\ 0 & \text { if } x \neq y\end{array}\right.$.

Theorem 2. Let $R$ be any fuzzy relation on $X$. Then $R$ is reflexive if and only if $R^{o p} \triangleleft_{\cdots_{*}} R \leq R$.

Proof. If $R$ is reflexive $I \leq R$ and $I=I^{o p} \leq R^{o p}$. Then by equation (13): $R=I \triangleleft_{\varkappa_{*}} R \geq R^{o p} \triangleleft_{\varkappa_{*}} R$.

Conversely, if $R^{o p} \triangleleft_{\rightsquigarrow_{*}} R \leq R$, for each $x \in X$, one can write:

$$
\begin{gathered}
R(x, x) \geq\left(R^{o p} \triangleleft_{\rightsquigarrow_{*}} R\right)(x, x)= \\
\inf _{w \in X}\left\{R^{o p}(x, w) \rightsquigarrow_{*} R(w, x)\right\}= \\
\inf _{w \in X}\left\{R(w, x) \rightsquigarrow_{*} R(w, x)\right\}=\inf _{w \in X}\{1\}=1 .
\end{gathered}
$$

Thus $I \leq R$ and $R$ is a reflexive relation.

Remark 2. In the case of a universe $X=\{x, y\}$ with two elements, $R$ is reflexive if and only if $R^{o p} \triangleleft_{\cdots_{*}} R=R$.

In fact, if $R$ reflexive:

$$
\begin{gathered}
\left(R^{o p} \triangleleft_{\rightsquigarrow_{*}} R\right)(x, y)=\inf _{w \in X}\left\{R^{o p}(x, w) \rightsquigarrow_{*} R(w, y)\right\}= \\
\inf _{w \in X}\left\{R(w, x) \rightsquigarrow_{*} R(w, y)\right\}= \\
\inf \left\{R(x, x) \rightsquigarrow_{*} R(x, y), R(y, x) \rightsquigarrow_{*} R(y, y)\right\}= \\
\inf \left\{1 \rightsquigarrow_{*} R(x, y), R(y, x) \rightsquigarrow_{*} 1\right\}=
\end{gathered}
$$

$\inf \{R(x, y), 1\}=R(x, y)$ and $R^{o p} \triangleleft_{\sim_{*}} R=R$. The converse is straightforward from Theorem 2.

Following the same argument as in the second part of the previous theorem, it is easy to prove that if $R^{o p} \triangleleft_{\rightsquigarrow_{*}} R \leq R^{o p}$ then $I \leq R^{o p}$, i.e. $I \leq R$.

Remark 3. If $R^{o p} \triangleleft_{w_{*}} R \leq R^{o p}$ then $R$ is reflexive. However, in this case, the converse does not hold as it is shown in the following simple example. 
Example. Consider the universe $X=\{x, y\}$ and the fuzzy reflexive relation in $[0,1]^{X \times X}$ given by $R=\left(\begin{array}{cc}1 & 0.3 \\ & \\ 0.1 & 1\end{array}\right)$. Then $R^{o p}=\left(\begin{array}{cc}1 & 0.1 \\ 0.3 & 1\end{array}\right)$ and from Remark 2, $R^{o p} \triangleleft_{\rightsquigarrow_{*}} R=R=\left(\begin{array}{cc}1 & 0.3 \\ 0.1 & 1\end{array}\right) \not \leq\left(\begin{array}{cc}1 & 0.1 \\ & \\ 0.3 & 1\end{array}\right)=R^{o p}$.

Remark 4. In the remain of this section, let us recall that $R_{C}$ denotes the *-preorder induced by $C$ by means of equation (4), i.e. $R_{C}(x, y)=C\left(\tilde{x}_{1}\right)(y)$, where $C=\varphi_{R}^{*}$.

Corollary 1. Let $R$ be any fuzzy relation on $X$. Then $R$ is reflexive if and only if $R_{C} \leq R^{o p}$.

Proof. This is straightforward from Theorem 2 and Lemma 3.

Corollary 2. Let $R$ be any fuzzy reflexive and symmetric relation on $X$, this is, a tolerance. Then $R_{C} \leq R$.

Proof. If $R$ is symmetric, $R^{o p}=R$. Then it is straightforward from Theorem 2 .

For the following result we will consider a negation such that $\alpha^{\prime}=\alpha \rightsquigarrow_{*} 0$. In this case, since $\left(R \rightsquigarrow_{*} Q\right)^{o p}=R^{o p} \rightsquigarrow_{*} Q^{o p}$, one gets: $\left(R^{o p}\right)^{\prime}=\left(R^{\prime}\right)^{o p}$.

Corollary 3. If $R$ is an irreflexive relation on $X$, i.e. $R(x, x)=0$ for all $x \in X$ then $R_{C} \leq R^{\prime}$.

Proof. If $R$ is irreflexive, $R \leq I^{\prime}$. Then by using the monotony property in the second argument of $\rightsquigarrow_{*}$ (property (8)), one gets:

$$
\begin{gathered}
\left(R^{o p} \triangleleft_{\rightsquigarrow_{*}} R\right)(x, y) \leq\left(R^{o p} \triangleleft_{\rightsquigarrow_{*}} I^{\prime}\right)(x, y)=\inf _{w \in X}\left\{R(x, w) \rightsquigarrow_{*} I^{\prime}(w, y)\right\} \leq \\
R(x, y) \rightsquigarrow_{*} I^{\prime}(y, y)=R(x, y) \rightsquigarrow_{*} 0=R^{\prime}(x, y)
\end{gathered}
$$

Observe that the previous results in this paragraph about reflexivity and symmetry do not make use of the t-norm $*$ since these properties do not depend on $*$.

Let us prove the following Lemma.

Lemma 4. Let $Q, R, S$ be fuzzy binary relations on $X$. Then

$$
Q \circ^{*} R \leq S \Longleftrightarrow R \leq Q^{o p} \triangleleft_{{ }_{*}} S
$$


Proof. $Q \circ^{*} R \leq S \Longleftrightarrow \sup _{y \in X}\{Q(x, y) * R(y, z)\} \leq S(x, z)$ for all $x, z \in X$ $\Longleftrightarrow Q(x, y) * R(y, z) \leq S(x, z)$ for all $x, y, z \in X \Longleftrightarrow R(y, z) \leq Q(x, y) \rightsquigarrow_{*}$ $S(x, z)$ for all $x, y, z \in X$ due to the adjointness property of residuated lattices.

Hence $Q \circ^{*} R \leq S \Longleftrightarrow R(y, z) \leq Q^{o p}(y, x) \rightsquigarrow_{*} S(x, z)$ for all $x, y, z \in X$.

Taking infimum in $x$, the previous statement is equivalent to $R(y, z) \leq$ $\inf _{x \in X}\left\{Q^{o p}(y, x) \rightsquigarrow_{*} S(x, z)\right\}$ for all $y, z \in X \Longleftrightarrow R(y, z) \leq\left(Q^{o p} \triangleleft_{\rightsquigarrow_{*}} S\right)(y, z)$ for all $y, z \in X \Longleftrightarrow R \leq Q^{o p} \triangleleft_{\rightsquigarrow_{*}} S$.

Let us recall that residuated lattices were introduced in 1939 by M. Ward and R.P. Dilworth (see [48]). This concept is more general than the one used by Bĕlohlávek in [2]. In particular, notice that the operation o* in Remark 5 is non-commutative.

Remark 5. Let us note that from Lemma 4 it is easy to obtain that the lattice of all fuzzy binary relations on $X,\left([0,1]^{X \times X}, \vee, \wedge, \circ^{*}, I, /, \backslash\right)$ is a residuated lattice, where $I$ is the identity relation, $I(x, y)=\left\{\begin{array}{ll}1 & \text { if } x=y \\ 0 & \text { if } x \neq y\end{array}\right.$, and the operations / and \are defined as follows

$$
\begin{gathered}
R / S=\left(S \triangleleft_{\rightsquigarrow_{*}} R^{o p}\right)^{o p} \\
R \backslash S=R^{o p} \triangleleft_{\rightsquigarrow_{*}} S
\end{gathered}
$$

The following result may be easily derived from previous Lemma and some properties in [4].

Theorem 3. Let $R$ be any fuzzy relation on $X$. Then $R$ is $*$-transitive if and only if $R \leq R^{o p} \triangleleft_{\varpi_{*}} R$.

Proof. Taking $R=S=Q$ in the previous Lemma, one has:

$$
R \circ^{*} R \leq R \Longleftrightarrow R \leq R^{o p} \triangleleft_{{ }_{*}} R
$$

Therefore: $\mathrm{R}$ is $*$-transitive $\Longleftrightarrow R \leq R^{o p} \triangleleft_{{ }_{*}} R$

Remark 6. Let us note that this is a result about the $*$-transitivity which does not involve explicitly the o operator. 
Corollary 4. Let $R$ be any fuzzy relation on $X$. Then $R$ is an $*$-preorder if and only if $R=R^{o p} \triangleleft_{\rightsquigarrow *} R$.

Proof. It is sufficient to consider Theorems 2 and 3

Remark 7. Using Lemma 3, the previous Corollary can be written as follows:

$$
R * \text { - preorder } \Longleftrightarrow R_{C}=R^{o p}
$$

Corollary 5. Let $R$ be any fuzzy reflexive, symmetric and $*$-transitive relation on $X$, this is, a fuzzy $*$-equivalence. Then $R_{C}=R$.

Proof. If $R$ is symmetric, $R^{o p}=R$. Then it is straightforward from Remark 7.

Remark 8. In this case ( $R$ fuzzy $*$-equivalence), one has $R_{C}=R \triangleleft_{\rightsquigarrow_{*}} R=R$.

Let us now show that the converse of Corollary 5 is also true.

Theorem 4. Let $R$ be any fuzzy relation, then $R$ is a fuzzy *-equivalence if and only if $R_{C}=R$.

Proof. We will prove only that if $R_{C}=R$ then $R$ is a fuzzy *-equivalence.

(i) Reflexivity: Recall that $R_{C}$ is always a fuzzy *-preorder. In particular $R_{C}$ is reflexive. Hence, if $R_{C}=R, R$ is also reflexive.

(ii) Symmetry: $R_{C}=R \Longleftrightarrow\left(R^{o p} \triangleleft_{\rightsquigarrow_{*}} R\right)^{o p}=R \Longleftrightarrow R^{o p}=R^{o p} \triangleleft_{\rightsquigarrow_{*}} R \Longrightarrow$ $R^{o p} \leq R^{o p} \triangleleft_{\rightsquigarrow_{*}} R$. By taking $Q=R, R=R^{o p}$ and $S=R$ in Lemma 4, one has: $R^{o p} \leq R^{o p} \triangleleft_{\rightsquigarrow_{*}} R$, i.e.,

$$
R \circ^{*} R^{o p} \leq R
$$

From previous inequality, one has $R(x, y) * R(z, y) \leq R(x, z)$ for all $x, y, z$ $\in X$.

Taking $y=x$, one has $R(z, x)=R(x, x) * R(z, x) \leq R(x, z)$ for all $x, z \in X$ due to the reflexivity of $R$.

Hence $R(x, z)=R(z, x)$ for all $x, z \in X$ and $R$ is symmetric.

(iii) $*$-transitivity: In the previous paragraph, we have proven that if $R_{C}=R$ then $R$ is symmetric and $R^{o p} \triangleleft_{\rightsquigarrow_{*}} R=R^{o p}$. Therefore $R^{o p} \triangleleft_{\rightsquigarrow_{*}} R=R$. From Theorem $3, R$ is $*$-transitive. 
Let us now discuss an application to fuzzy relational equations. It is known that the fuzzy relational equation $R \circ^{*} X=S(R \neq S)$ has a solution if and only if $X_{0}=R^{o p} \triangleleft_{\rightsquigarrow_{*}} S$ is a solution and moreover it corresponds to the greatest solution $([21,36])$.

Corollary 6. The fuzzy relational equation $R \circ^{*} X=R$ has non-trivial solutions if and only if $X_{0}=R^{o p} \triangleleft_{\rightsquigarrow_{*}} R=\left(R_{C}\right)^{o p}$ is a non-trivial solution. In this case it is the greatest solution.

Proof. It is enough to consider the case $R=S$, the previous paragraph and Lemma 3.

Notice that in the case $R=S$ the identity $X=I$ is always a trivial solution of the relational equation $R \circ^{*} X=R$.

Remark 9. $X_{0}$ can be seen both as the inverse of the fuzzy relation $R_{C}$ and as the erosion of $R$ with itself.

\section{Embedding fuzzy closing morphological operators in the classes of fuzzy consequence operators, fuzzy preorders and closure sys- tems}

First, we prove the following lemma in order to obtain some interesting relationships which involves fuzzy closing morphological operators and fuzzy consequence operators induced by means of (1) and (14) respectively when the relation is a $*$-preorder.

Lemma 5. Let $C$ be any *-coherent operator (satisfying (3), not necessarily fuzzy consequence operator). Then $\theta^{\prime *} \circ \tilde{\theta}^{\prime}(C) \subset C$.

Proof. If $C$ satisfies (3), one gets:

$$
\begin{gathered}
\theta^{\prime *} \circ \tilde{\theta}^{\prime}(C)(\mu)(x)=C_{\tilde{\theta}^{\prime}(C)}^{*}(\mu)(x)= \\
\sup _{w \in X}\left\{\mu(w) * \tilde{\theta}^{\prime}(C)(w, x)\right\}= \\
\quad \sup _{w \in X}\left\{\mu(w) * R_{C}(w, x)\right\}= \\
\sup _{w \in X}\left\{\mu(w) * C\left(\varphi_{w}\right)(x)\right\} \leq \\
\sup _{w \in X}\{C(\mu)(x)\}=C(\mu)(x)
\end{gathered}
$$


Therefore $\theta^{\prime} * \tilde{\theta}^{\prime}(C) \subset C$.

Theorem 5. Let $R$ be any fuzzy relation and let $*$ denote a $t$-norm. If $R$ is $a *$-preorder then $\varphi_{R}^{*} \in \Omega_{p}^{*}$, this is, $\varphi_{R}^{*}$ is induced by a*-preorder by means of (1).

Proof. Assume that $R$ is $*$-preorder and prove that $\theta^{*} \circ \tilde{\theta}\left(\varphi_{R}^{*}\right)=\varphi_{R}^{*}$. From Theorem $1, \varphi_{R}^{*}$ is a $*$-coherent operator, then $\theta^{*} \circ \tilde{\theta}\left(\varphi_{R}^{*}\right) \subset \varphi_{R}^{*}$ by Lemma 5 .

In addition, one can write

$$
\begin{gathered}
\varphi_{R}^{*}(\mu)(x)=\inf _{w \in X}\left\{R(w, x) \rightsquigarrow_{*} C_{R^{o p}}^{*}(\mu)(w)\right\} \leq \\
R(x, x) \rightsquigarrow_{*} C_{R^{o p}}^{*}(\mu)(x)=1 \rightsquigarrow_{*} C_{R^{o p}}^{*}(\mu)(x)= \\
C_{R^{o p}}^{*}(\mu)(x)
\end{gathered}
$$

From Corollary 4 , if $\mathrm{R} *$-preorder one gets

$$
R_{C}=\tilde{\theta}\left(\varphi_{R}^{*}\right)=R^{o p}
$$

Therefore

$$
\begin{gathered}
\varphi_{R}^{*} \subset C_{R^{o p}}^{*}=\theta^{*}\left(R^{o p}\right)= \\
\theta^{*}\left(\tilde{\theta}\left(\varphi_{R}^{*}\right)\right)=\theta^{*} \circ \tilde{\theta}\left(\varphi_{R}^{*}\right)
\end{gathered}
$$

Let us note that if $R$ is a $*$-preorder then $\varphi_{R}^{*} \in \Omega_{p}^{*}$ and there exists a *preorder $S$ such that $\varphi_{R}^{*}=C_{S}^{*}$. Thus

$$
\tilde{\theta}\left(\varphi_{R}^{*}\right)=\tilde{\theta}\left(C_{S}^{*}\right)=\tilde{\theta} \circ \theta^{*}(S)=S
$$

that is

$$
R_{C}=S
$$

From Corollary 4, $R_{C}=R^{o p}$ and $S=R^{o p}$.

From the latter, the following corollary is straightforward.

Corollary 7. If $R$ is a *-preorder then $\varphi_{R}^{*}=C_{R^{o p}}^{*}$.

For an equivalence relation $R$, as $R^{o p}=R$ we obtain immediately the following result.

Corollary 8. If $R$ is a *-equivalence then $\varphi_{R}^{*}=C_{R}^{*}$.

It is easy to obtain related results for non-transitive fuzzy relations. If $R$ is only reflexive: 
Theorem 6. If $R$ is reflexive then $\varphi_{R}^{*} \leq C_{R^{o p}}^{*}$.

Proof. If $R$ is reflexive then

$$
\begin{gathered}
\varphi_{R}^{*}(\mu)(x)=\inf _{t \in X}\left\{R(t, x) \rightsquigarrow_{*} \sup _{w \in X}\{R(t, w) * \mu(w)\}\right\} \leq \\
R(x, x) \rightsquigarrow_{*} \sup _{w \in X}\{R(x, w) * \mu(w)\}
\end{gathered}
$$

By using the reflexivity property, one gets:

$$
\begin{gathered}
\varphi_{R}^{*}(\mu)(x) \leq 1 \rightsquigarrow_{*} \sup _{w \in X}\{R(x, w) * \mu(w)\}= \\
\sup _{w \in X}\{\mu(w) * R(x, w)\}=C_{R^{o p}}^{*}(\mu)(x)
\end{gathered}
$$

If $R$ is a tolerance:

Corollary 9. If $R$ is reflexive and symmetric then $\varphi_{R}^{*} \leq C_{R}^{*}$.

Let us now recall that $\Gamma^{*}$ denotes the set of all $*$-preorder and $\Omega_{p}^{*}=$ $\left\{C \in \Omega \mid \exists R \in \Gamma^{*}, C=C_{R}^{*}\right\}$ indicates the set of all fuzzy consequence operators that are induced by a preorder by means of equation (1). Let us now consider the following operations on the family $\Omega_{p}^{*}([23])$ in order to obtain an isomorphism between both families $\Gamma^{*}$ and $\Omega_{p}^{*}$.

Let $\left(C_{R_{i}}^{*}\right)_{i \in I}$ be an arbitrary family of operators in $\Omega_{p}^{*}$, we define the *intersection of $\left(C_{R_{i}}^{*}\right)_{i \in I}$ by $\bigwedge_{i \in I}^{*} C_{R_{i}}^{*}=C_{\bigcap_{i \in I}^{*} R_{i}}^{*}$, i.e., the $*$-intersection of any family $\left(C_{R_{i}}^{*}\right)_{i \in I}$ of operators is the operator induced by the intersection of all preorders that have induced this family. It is well defined since $\Gamma^{*}$ is closed under arbitrary intersections.

We define the $*$-union of $\left(C_{R_{i}}^{*}\right)_{i \in I}$ as follows:

$$
\bigsqcup_{i \in I}^{*} C_{R_{i}}^{*}=\bigwedge^{*}\left\{C \in \Omega_{p}^{*} \mid C \supset \bigcup_{i \in I} C_{R_{i}}^{*}\right\}
$$

this is, the $*$-union of any family of operators $\left(C_{R_{i}}^{*}\right)_{i \in I}$ is the previous intersection of all operators in $\Omega_{p}^{*}$ that contains the supremum of the family $\left(C_{R_{i}}^{*}\right)_{i \in I}$.

In the previous operations we have included the $*$ symbol since they depend on the t-norm $*$. 
The function $\theta^{*}\left(\theta^{*}(R)=C_{R}^{*}\right)$ is a lattice isomorphism between complete lattices $\left(\Gamma^{*}, \sqcup^{*}, \wedge\right)$ and $\left(\Omega_{p}^{*}, \sqcup^{*}, \wedge^{*}\right)$. In particular for two fuzzy $*$-preorders one gets:

$$
\begin{aligned}
& \theta^{*}\left(R_{1} \sqcup^{*} R_{2}\right)=C_{R_{1} \sqcup^{*} R_{2}}^{*}=C_{R_{1}}^{*} \sqcup^{*} C_{R_{2}}^{*}=\theta^{*}\left(R_{1}\right) \sqcup^{*} \theta^{*}\left(R_{2}\right) \text { and } \\
& \theta^{*}\left(R_{1} \wedge R_{2}\right)=C_{R_{1} \wedge R_{2}}^{*}=C_{R_{1}}^{*} \wedge^{*} C_{R_{2}}^{*}=\theta^{*}\left(R_{1}\right) \wedge^{*} \theta^{*}\left(R_{2}\right) .
\end{aligned}
$$

In order to embed the fuzzy preorders in the structure of fuzzy consequence operators by using a monomorphism between lattices, we proved that the restriction of $\sqcup^{\prime}$ defined in $\Omega$ to family $\Omega_{p}^{*}$ is precisely $\sqcup^{*}$, that is, $\sqcup^{\prime} \mid \Omega_{p}^{*}=\sqcup^{*}$. Consequently, $\left(\Omega_{p}^{*}, \sqcup^{*}, \wedge\right)$ is a complete $\sqcup^{\prime}$-sublattice of complete lattice $\left(\Omega, \sqcup^{\prime}, \wedge\right)$. Then $\left(\Gamma^{*}, \sqcup^{*}, \wedge\right) \cong\left(\Omega_{p}^{*}, \sqcup^{*}, \wedge^{*}\right) \leq\left(\Omega, \sqcup^{\prime}, \wedge\right)$.

Let us now connect the previous classes of fuzzy operators and preorders with the structures of fuzzy closure and co-closure systems. We denote by $C C S_{p}^{*}(X)$ the image set of family $\Gamma^{*}$ by function $\Im^{*}$, this is, $C C S_{p}^{*}(X)=$ $\Im^{*}\left(\Gamma^{*}\right)=\left\{S \in C C S(X) \mid \exists R \in \Gamma^{*}, \Im^{*}(R)=S\right\} \subset C C S(X) \subset C S(X)$.

Since $\Im^{*} \mid \Gamma^{*}$ is a one to one function, $\Im^{*}\left(\Gamma^{*}\right)=\{S \in C C S(X) \mid$ $\left.\Im^{*}\left(I^{*}(S)\right)=S\right\}$. Moreover as $\theta^{*}$ is a lattice isomorphism between $\Gamma^{*}$ and $\Omega_{p}^{*}$ such that $\Im^{*}=\alpha \circ \theta^{*}$, one gets to the equality $\alpha\left(\Omega_{p}^{*}\right)=\Im^{*}\left(\Gamma^{*}\right)$, hence $\left(C C S_{p}^{*}(X), \wedge\right)$ is a complete $\wedge$-sublattice of complete semilattice $(C S(X), \wedge)$.

Therefore the classes of fuzzy preorders and fuzzy consequence operators are embedded in the structure of fuzzy closure systems: $\left(\Gamma^{*}, \sqcup^{*}\right) \cong$ $\left(\Omega_{p}^{*}, \sqcup^{*}\right) \cong{ }^{d}\left(C C S_{p}^{*}(X), \wedge\right)$.

To finish the demonstration, we will embed the class of fuzzy closing morphological operators in the previous lattices structures.

Let us consider the class of fuzzy closing morphological operators induced by a $*$-preorder by means of (14): $\Omega_{c}^{*}=\left\{\varphi \in \Omega \mid \exists R \in \Gamma^{*}, \varphi=\varphi_{R^{o p}}^{*}\right\}$ and let us prove some preliminary results.

Remark 10. Given a fuzzy relation $R$ :

$$
R \in \Gamma^{*} \Longleftrightarrow R^{o p} \in \Gamma^{*}
$$

Indeed, $R$ is $*$-transitive $\Longleftrightarrow R \circ^{*} R \subset R \Longleftrightarrow\left(R \circ^{*} R\right)^{o p} \subset R^{o p} \Longleftrightarrow$ $R^{o p} \circ^{*} R^{o p} \subset R^{o p} \Longleftrightarrow R^{o p}$ is $*$-transitive. From the latter, it is obvious that $R$ reflexive $\Longleftrightarrow R^{o p}$ reflexive. 
Remark 11. Given any family of fuzzy relations $\left(R_{i}\right)_{i \in I}$, it is easy to prove that $\left(\bigcap_{i \in I} R_{i}\right)^{o p}=\bigcap_{i \in I} R_{i}^{o p}([4])$.

Lemma 6. Given $R_{1}, R_{2} \in \Gamma^{*}$, the following equality holds:

$$
\left(R_{1} \sqcup^{*} R_{2}\right)^{o p}=R_{1}^{o p} \sqcup^{*} R_{2}^{o p}
$$

Proof. Using remarks 10 and 11, one gets:

$$
\begin{gathered}
\left(R_{1} \sqcup^{*} R_{2}\right)^{o p}=\left(\bigcap_{i \in I}\left\{R_{i} \in \Gamma^{*} \mid R_{i} \supset R_{1} \vee R_{2}\right\}\right)^{o p}= \\
\bigcap_{i \in I}\left\{R_{i}^{o p} \in \Gamma^{*} \mid R_{i} \supset R_{1} \vee R_{2}\right\}= \\
\bigcap_{i \in I}\left\{R_{i} \in \Gamma^{*} \mid R_{i}^{o p} \supset R_{1} \vee R_{2}\right\}= \\
\bigcap_{i \in I}\left\{R_{i} \in \Gamma^{*} \mid R_{i} \supset\left(R_{1} \vee R_{2}\right)^{o p}\right\}= \\
\bigcap_{i \in I}\left\{R_{i} \in \Gamma^{*} \mid R_{i} \supset R_{1}^{o p} \vee R_{2}^{o p}\right\}=R_{1}^{o p} \sqcup^{*} R_{2}^{o p}
\end{gathered}
$$

Let us now define the following new operations on the family $\Omega_{c}^{*}$. For any arbitrary family of operators $\left(\varphi_{R_{i}^{o p}}^{*}\right)_{i \in I}$ in $\Omega_{c}^{*}$, the $*$-intersection $\left(\varphi_{R_{i}^{o p}}^{*}\right)_{i \in I}$ is given by $\bigwedge_{i \in I}^{*} \varphi_{R_{i}^{o p}}^{*}=\varphi_{\bigcap_{i \in I}^{*} R_{i}{ }^{o p}}$ and the $*$-union of $\left(\varphi_{R_{i}^{o p}}^{*}\right)_{i \in I}$ is given by $\bigsqcup_{i \in I}^{*} \varphi_{R_{i}^{o p}}^{*}=\bigwedge^{*}\left\{C \in \Omega_{c}^{*} \mid C \supset \bigcup_{i \in I} \varphi_{R_{i}^{o}}^{*}\right\}$. The latter are well-defined since $\Gamma^{*}$ is closed under arbitrary intersections.

Consequently, $\left(\Omega_{c}^{*}, \sqcup^{*}, \wedge^{*}\right)$ is a complete lattice. Moreover, it is analogous to prove that the restriction $\sqcup^{\prime}$ defined in $\Omega$ to family $\Omega_{c}^{*}$ is precisely $\sqcup^{*}$, i.e., $\sqcup^{\prime} \mid \Omega_{c}^{*}=\sqcup^{*}$. Then $\left(\Omega_{c}^{*}, \sqcup^{*}, \wedge^{*}\right)$ is a complete $\sqcup^{\prime}$-sublattice of complete lattice $\left(\Omega, \sqcup^{\prime}, \wedge\right)$ and $\left(\Omega_{c}^{*}, \sqcup^{*}, \wedge^{*}\right) \leq\left(\Omega, \sqcup^{\prime}, \wedge\right)$.

Let us introduce another function $\gamma^{*}: \Gamma^{*} \longrightarrow \Omega_{c}^{*}$ given by $\gamma^{*}(R)=\varphi_{R^{o p}}^{*}$.

Theorem 7. The function $\gamma^{*}$ is an isomorphism between complete lattices $\left(\Gamma^{*}, \sqcup^{*}, \wedge\right)$ and $\left(\Omega_{c}^{*}, \sqcup^{*}, \wedge^{*}\right)$. 
Proof. It is enough to prove that $\gamma^{*}$ is an isotone bijection with isotone inverse.

Assume that $\gamma^{*}\left(R_{1}\right)=\gamma^{*}\left(R_{2}\right) \Longrightarrow \varphi_{R_{1}^{o p}}^{*}=\varphi_{R_{2}^{o p}}^{*} \Longrightarrow C_{R_{1}}^{*}=C_{R_{2}}^{*} \Longrightarrow$ $\theta^{*}\left(R_{1}\right)=\theta^{*}\left(R_{2}\right)$. As $\theta^{*}$ is one-one, $R_{1}=R_{2}$ and so is $\gamma^{*}$.

Given $\varphi_{R}^{*} \in \Omega_{c}^{*}$, let us take $R^{o p} \in \Gamma^{*}$ and $\gamma^{*}(R)=\varphi_{\left(R^{o p}\right)^{o p}}^{*}=\varphi_{R}^{*}$ and $\gamma^{*}$ is onto and it is a bijection.

Note that obviously $R_{1} \subset R_{2} \Longleftrightarrow R_{1}^{o p} \subset R_{2}^{o p}$ and let us recall that $\theta^{*}$ and $\tilde{\theta}$ are isotones functions. Then suppose that $R_{1} \subset R_{2} \Longrightarrow C_{R_{1}}^{*} \subset C_{R_{2}}^{*} \Longrightarrow$ $\varphi_{R_{1}^{o p}}^{*} \subset \varphi_{R_{2}^{o p}}^{*} \Longrightarrow \gamma^{*}\left(R_{1}\right) \subset \gamma^{*}\left(R_{2}\right)$ and $\gamma^{*}$ is isotone. Next, suppose that $\varphi_{R_{1}}^{*} \subset \varphi_{R_{2}}^{*} \Longrightarrow C_{R_{1}^{o p}}^{*} \subset C_{R_{2}^{o p}}^{*} \Longrightarrow R_{1}^{o p} \subset R_{2}^{o p} \Longrightarrow R_{1} \subset R_{2}$ and $\gamma^{*-1}$ is also isotone. The latter completes the proof.

In consequence, we have

$$
\left(\Gamma^{*}, \sqcup^{*}, \wedge\right) \cong\left(\Omega_{c}^{*}, \sqcup^{*}, \wedge^{*}\right) \leq\left(\Omega, \sqcup^{\prime}, \wedge\right)
$$

Finally, we can proceed as in the case of $\Omega_{p}^{*}$ with respect to fuzzy closure systems to obtain

$$
\left(\Gamma^{*}, \sqcup^{*}\right) \cong\left(\Omega_{c}^{*}, \sqcup^{*}\right) \cong{ }^{d}\left(C C S_{p}^{*}(X), \wedge\right)
$$

Remark 12. We can obtain a commutative diagram analogous to the one in $[23]$ replacing $\Omega_{p}^{*}$ with $\Omega_{c}^{*} ; \theta^{*}$ with $\gamma^{*}$; and $\tilde{\theta}$ with $\gamma^{*-1}$.

\section{Conclusions and future works}

In this paper, we have connected the class of fuzzy consequence operators used in the context of approximate reasoning with the class of fuzzy closing operators used in image processing acting as morphological filters.

Given a fuzzy relation $R$ used as the structuring element, we have shown in Theorem 1 that the fuzzy closing operator $C=\varphi_{R}^{*}$ induced by such relation is always a $*$-coherent fuzzy consequence operator. Therefore such operator $C$ induces always a preorder $R_{C}$.

We have also obtained in Lemma 3 the following representation of the induced relation:

$$
R_{C}=\left(R^{o p} \triangleleft_{\rightsquigarrow_{*}} R\right)^{o p}
$$


Theorem 1 and Lemma 3 have been the key for obtaining the subsequent properties that we enumerate below.

We have shown some characterizations of the $*$-reflexivity and $*$-transitivity of a fuzzy relation $R$. In consequence, we have characterized when a fuzzy relation is an $*$ - preorder or $*$-indistinguishability.

1. $R$ is reflexive if and only if $R^{o p} \triangleleft_{\rightsquigarrow_{*}} R \leq R$ (Theorem 2)

2. $R$ is reflexive if and only if $R_{C} \leq R^{o p}$ (Corollary 1 )

3. $R$ is $*$-transitive if and only if $R \leq R^{o p} \triangleleft_{\rightsquigarrow_{*}} R$ (Theorem 3)

4. $R$ is an *-preorder if and only if $R=R^{o p} \triangleleft_{\rightsquigarrow_{*}} R$ (Corollary 4 )

It should be noticed that the previous characterization about the transitivity does not involve the o operator explicitly because we have characterized the transitivity of a relation $R$ using the operator $\triangleleft$ instead of $\circ$.

In addition, we have demonstrated some properties of the preorder $R_{C}$ induced by the fuzzy closing operator $C=\varphi_{R}^{*}$.

5. $R$ is a fuzzy $*$-preorder if and only if $R_{C}=R^{o p}$ (Remark 7)

6. $R$ is a fuzzy $*$-indistinguishability if and only if $R_{C}=R$ (Theorem 4)

7. If $R$ is $*$-preorder then $\varphi_{R}^{*}=C_{R^{o p}}^{*}($ Corollary 7$)$

Let us note that the previous result shows that if $R$ is a $*$ - preorder then the fuzzy closing morphological operator induced by $R$ by means of equation (14) is also induced by $R$ as fuzzy consequence operator by means of equation (1).

8. If $R$ is $*$-indistinguishability then $\varphi_{R}^{*}=C_{R}^{*}$ (Corollary 8)

Finally, classes of fuzzy closing morphological, fuzzy consequence operators, fuzzy preorders and fuzzy closure systems have been connected in the framework of complete lattices as isomorphic structures as a consequence of Theorem 7 .

In future works we will use these connections to translate the well known properties from the field of approximate reasoning to the one of fuzzy relational systems. In particular we will explore their applications to image processing. 


\section{Acknowledgements}

This research is partially supported by MICINN (Spain) under the project FIS2008-06335-C02-02 and Gobierno de Navarra (Spain) under the project $2008 / 2301$.

We thank the anonymous referees for their valuable suggestions that have helped to improve the content and the presentation of this paper.

\section{References}

[1] H. Bandemer, W. Näther, Fuzzy Data Analysis. Theoria and Decision Library, Series B: Mathematical and Statistical Methods, Kluwer Academic Publishers, Dordrecht, 1992.

[2] R. Bĕlohlávek. Fuzzy closure operators. Journal of Mathematical Analysis and Appl. Vol 262, pp. 473-489, 2001.

[3] R. Bĕlohlávek. Fuzzy closure operators II. Soft Computing Vol 7. No 1, pp, 53-64, 2002.

[4] R. Bĕlohlávek. Fuzzy relational systems. Foundations and principles (IFSR International Series on Systems Science and Engineering, Vol 20, Kluwer Academic/Plenum Plubishers, New York, 2002)

[5] G. Birkhoff, Lattice Theory (American Mathematical Society Colloquium Publications, Vol 25, 1967).

[6] I. Bloch. Duality vs adjunction for fuzzy mathematical morphology and general form of fuzzy erosions and dilations. Fuzzy sets and systems Vol 160. pp, 1858-1867, 2009.

[7] I. Bloch. Fuzzy and Bipolar Mathematical Morphology, Applications in Spatial Reasoning. Lecture Notes in Computer Science Vol 5590, pp. 1-13. Springer. 2009

[8] I. Bloch. Lattices of fuzzy sets and bipolar fuzzy sets, and mathematical morphology. Information Sciences Vol 181, pp, 2002-2015, 2011.

[9] I. Bloch. Triangular Norms as a Tool for Constructing Fuzzy Mathematical Morphologies. Proc. of the Internat. Workshop on Mathematical Morphology and its Applications to Signal Processing, pp, 157-161. Barcelona, Spain. 1993. 
[10] I. Bloch, H. Maître. Fuzzy Mathematical Morphologies: a comparative study. Pattern Recognition Vol 28. No 9, pp, 1341-1387, 1995.

[11] J.L. Castro. Fuzzy logics as families of bivaluated logics. Fuzzy sets and systems 64, pp. 321-332, 1994.

[12] J.L. Castro, M. Delgado, E. Trillas. Inducing Implication Relations. International Journal of Approximate Reasoning. Vol 10, pp. 235-250, 1994.

[13] J.L. Castro, F. Klawonn. Similarity in Fuzzy Reasoning. Mathware and Soft Computing, Vol. 2, pp. 157-228, 1996.

[14] J.L. Castro and E. Trillas, Sobre preórdenes y operadores de consecuencias de Tarski, Theoria Second period, Course 1988-1989, Vol 11, pp. 419-425, 1989 (in Spanish)

[15] J.L. Castro, E. Trillas. Tarski's fuzzy consequences. Proc. of the Internat. Fuzzy Engineering Symp'91. Vol.1, pp. 70-81, 1991.

[16] B. De Baets. Generalized idempotence in fuzzy mathematical morphology, in Fuzzy Techniques in Image Processing (E. Kerre and M. Nachtegael, eds.), Studies in Fuzziness and Soft Computing Physica-Verlag Vol 52, pp, 58-75, 2000.

[17] B. De Baets. Idempotent closing and opening operations in fuzzy mathematical morphology. Proc. of the Joint Third Internat. Symposium on Uncertainty Modeling and Analysis and Annual Conference of the North American Fuzzy Information Processing Society. (College Park, MD, USA), IEEE Computer Society Press, Los Alamitos, pp. 228-233, 1995.

[18] B. De Baets, E. Kerre, M. Gupta. The fundamentals of fuzzy mathematical morphology. Part 1: Basic concepts. International Journal of General Systems. Vol 23, pp. 155-171, 1994

[19] B. De Baets, E. Kerre, M. Gupta. The fundamentals of fuzzy mathematical morphology. Part 2: Idempotence,convexity and decomposition. International Journal of General Systems. Vol 23, pp. 307-322, 1995

[20] T.Q. Deng, H.J.A.M. Heijmans. Grey-scale morphology based on fuzzy logic, Journal of mathematical Imaging and Vision 16 (2002) 155-171. 
[21] A. Di Nola, A. Lettieri. Relation equations in residuated lattices. Rendiconti del Circolo matematico di Palermo, s.II, XXXVIII, pp. 246-256, 1989.

[22] J. Elorza, P. Burillo. On the relation between fuzzy preorders and fuzzy consequence operators. International Journal of Uncertainty, Fuzziness and Knowledge-Based Systems. Vol.7. No 3, pp. 219-234, 1999.

[23] J. Elorza, P. Burillo. Connecting fuzzy preorders, fuzzy consequence operators and fuzzy closure and co-closure systems. Fuzzy sets and systems. Vol.139. No 3, pp. 601-613, 2003.

[24] J. Elorza, R. Fuentes-González, J. Bragard, P. Burillo. Fuzzy Closing Operators and their Coherence as Fuzzy Consequence Operators Proc. of the 14th Spanish Conference on Fuzzy Logic and Technologies (ESTYLF 2008), pp. 221-227, 2008.

[25] J. Elorza, R. Fuentes-González, J. Bragard, P. Burillo. On the relationship between Fuzzy preorders, Fuzzy Closing Morphological Operators and Fuzzy Consequence Operators Proc. of the 15th Spanish Conference on Fuzzy Logic and Technologies (ESTYLF 2010), pp. 133-138, 2010.

[26] F. Esteva, P. García, L. Godo, R.O. Rodríguez. Fuzzy Approximation Relations, Modal Structures and Possibilistic Logic. Mathware and Soft Computing, Vol. V, n 2-3, pp. 151-166, 1998.

[27] R. Fuentes-González, P. Burillo, N. Frago. Técnicas de Morfología Matemática en el tratamiento de Sistemas Relacionales Difusos Proc. of the 11th Spanish Conference on Fuzzy Logic and Technologies (ESTYLF 2002), pp. 321-326, 2002. (in Spanish)

[28] L. Garmendia, J. Recasens. Approximation of Proximities by Aggregating T-indistinguishability Operators. Mathware and Soft Computing, Vol. 14, pp. 171-181, 2007.

[29] G. Gerla. Comparing fuzzy and crisp deduction systems, Fuzzy Sets and Systems 67, pp. 317-328. 1994

[30] H.J.A.M. Heijmans. Morphological Image Operators. Academic Press. 1994. 
[31] H.J.A.M. Heijmans, C. Ronse. The Algebraic Basis of Mathematical Morphology. Part I: Dilations and erosions. Computer Vision, Graphics and Image Processing Vol 50. No 3, pp, 245-295, 1990.

[32] P. Maragos. Lattice image processing: a unification of morphological and fuzzy algebraic systems Journal of Mathematical Imaging and Vision Vol 22, pp, 333-353, 2005.

[33] G. Matheron. Random Sets and Integral Geometry. Wiley. NY. 1975

[34] M. Nachtegael, P. Sussner, T. Melange, E. Kerre. On the role of complete lattices in mathematical morphology: From tool to uncertainty model. Information Sciences Vol 181, pp, 1971-1988, 2011.

[35] J. Pavelka. On Fuzzy Logic I. Zeitschr. f. Math. Logik und Grundlagen d. Math. Bd.25, pp. 45-52, 1979.

[36] K. Peeva, Y. Kyosev. Algorithm for solving max-product fuzzy relational equations. Soft Computing Vol 11. No 7, pp, 593-605, 2007.

[37] A.M. Radzikowska, E.E. Kerre. Characterisation of main classes of fuzzy relations using fuzzy modal operators. Fuzzy sets and systems. Vol.152. No 2, pp. 223-247, 2005.

[38] R.O. Rodríguez, F. Esteva, P. García, L. Godo. On Implicative Closure Operators in Approximate Reasoning, Internat. Journal of Approximate Reasoning. Vol.33, pp. 159-184, 2003.

[39] C. Ronse, H.J.A.M. Heijmans. The Algebraic Basis of Mathematical Morphology. Part II: Openings and Closings. Computer Vision, Graphics and Image Processing Vol 54. No 1, pp, 74-97, 1991.

[40] J. Serra. Image Analysis and Mathematical Morphology. Academic Press. Vol I (fourth printing, 1990) and Vol II (second printing, 1992).

[41] D. Sinha, E.R. Dougherty. Fuzzification of set inclusion: theory and applications. Fuzzy sets and systems. Vol.55, pp. 15-42, 1993.

[42] P. Soille. Morphological Image Analysis. Principles and Applications. Springer. 1999. 
[43] P. Sussner, M.E. Valle. Classification of fuzzy mathematical morphologies based on concepts of inclusion measure and duality. Journal of Mathematical Imaging and Vision Vol 32. No 2, pp, 139-159, 2008.

[44] A. Tarski. Logic, semantics, metamathematics. Clarendon Press, Oxford, 1956.

[45] E. Trillas, C. Alsina and J.M. Terricabras. Introducción a la lógica borrosa, Ariel, 1995. (in Spanish)

[46] M.E. Valle, P. Sussner. A general framework for fuzzy morphological associative memories. Fuzzy sets and systems. Vol.159. No 7, pp. 747$768,2008$.

[47] M. Ward. The closure operators of a lattice. Annals of Mathematics. Vol.43. N.2, pp. 191-196, 1940.

[48] M. Ward and R.P. Dilworth. Residuated lattices. Transactions of the American Mathematical Society. Vol.45. N.3, pp. 335-354, 1939.

[49] L.A. Zadeh, Fuzzy sets, Information and Control 8, pp. 338-353, 1965.

[50] L.A. Zadeh, Similarity Relations and Fuzzy Orderings, Information Sciences 3, pp. 177-200, 1971. 